\title{
Diagnosis and management of AIDS-related diarrhea
}

\author{
JOHN F JOHANSON MD MSc Epid
}

JF JOHANSON. Diagnosis and management of AIDS-related diarrhea. Can J Gastroenterol 1996;10(7):461-468. The spectrum of illness associated with the acquired immunodeficiency syndrome (AIDS) has been increasing since the initial description in 1981. While virtually all organ systems may be affected, the gastrointestinal tract appears to be a major target. Diarrhea is the most common symptom, affecting up to half of all AIDS patients during the course of their disease. Although diarrhea occurs frequently, its optimal management remains controversial. An extensive evaluation including stool studies and endoscopic biopsies of both the colon and small intestine has been widely recommended to identify all potential pathogenic organisms. An alternative approach is a more limited evaluation consisting of stool and blood cultures followed by symptomatic treatment with antidiarrheal agents if no specific organisms are identified. The clinical presentation of the most common opportunistic pathogens are reviewed, including several recently discovered organisms. Recommendations for treatment are followed by a brief discussion of management strategies used to care for patients with AIDS-related diarrhea.

Key Words: AIDS, Diarrhea, Gastrointestinal tract, Treatment

\section{Diagnostic et traitement de la diarrhée associée au SIDA}

RÉSUMÉ : L'éventail des maladies associées au syndrome de l'immunodéficience acquise (SIDA) s'élargit constamment depuis la description des premiers cas en 1981. Pour ainsi dire tous les systèmes de l'organisme peuvent être touchés, mais le tractus digestif semble être une cible de choix. La diarrhée est le symptôme le plus courant, affectant jusqu'à la moitié de tous les patients sidéens au cours de la maladie. La diarrhée est fréquente, mais son traitement demeure controversé. Une évaluation approfondie y compris des analyses de selles et des biopsies par endoscopie du côlon et du grêle ont été très recommandées afin d'identifier tous les organismes pathogènes potentiels. Une autre approche consiste à se restreindre à des hémocultures et à des cultures de selles, et d'administrer un traitement selon les symptômes, au moyen d'agents anti-diarrhéiques si aucun organisme pathogène n'est identifié. Le tableau clinique des infections causées par les organismes pathogènes les plus courants est passé en revue, y compris celui des infections causées par les organismes les plus récemment découverts. Les recommandations thérapeutiques sont suivies d'une brève discussion sur les stratégies de prise en charge des patients souffrant de diarrhée associée au SIDA.
$\mathrm{T}$ he spectrum of illness associated with the acquired immunodeficiency syndrome (AIDS) has been increasing since the initial description in 1981. While virtually all organ systems may be affected, the gastrointestinal tract appears to be a major target. Approximately $50 \%$ of all AIDS patients will develop diarrhea that is typically chronic, watery, nonbloody, and often associated with malnutrition and progressive weight loss (1-9). The underlying risk factor for acquiring AIDS appears to play an influential role in the development of diarrhea. Up to $80 \%$ of homosexual or bisexual patients develop diarrheal disorders compared with fewer than $50 \%$ of patients who have other risk factors for AIDS a statistically significant difference $(10,11)$.

\section{ORGANISMS}

The integrity of the host's immune system is clearly important in protecting against infectious pathogens (12). Originally it was thought that immune dysfunction predisposed the gut to bacterial colonization which, in turn, induced malabsorption and chronic diarrhea (5). Although bacterial overgrowth may have some etiological influence, it appears that dysfunction of cell-mediated immunity, a direct result of infection by the AIDS virus, is responsible for the development of opportunistic infections, which leads to diarrhea. Moreover, as immune function worsens, the prevalence and severity of diarrhea increases. Thus, the onset of diarrhea may act as a marker of deteriorating immune func- 
tion $(11,13)$. Opportunistic infections have been identified in $50 \%$ to $85 \%$ of diarrheal episodes, and the majority of causative agents fall into three main categories: protozoa, viruses and bacteria.

Protozoa - Cryptosporidium: Cryptosporidium is one of the most common organisms that causes diarrhea. It occurs in $10 \%$ to $20 \%$ of patients with AIDS-related diarrhea in the United States. It has been found in up to $55 \%$ of patients with AIDS in developing countries. Cryptosporidium belongs to the class sporozoa as do the majority of other protozoa that afflict AIDS patients (14). Cryptosporidia are small (4 to $6 \mu \mathrm{m})$ spherical organisms that, in immunocompromised patients, cause a debilitating diarrheal illness characterized by large volume, watery diarrhea (typically 1 to $2 \mathrm{~L} /$ day), cramping periumbilical abdominal pain and a variety of constitutional symptoms. Patients who develop cryptosporidial infections typically have CD4 counts less than 100 cells $/ \mathrm{mm}^{3}$ and often their counts are less than 50 cells/ $\mathrm{mm}^{3}$. Patients with CD4 counts greater than 180 cells $/ \mathrm{mm}^{3}$ usually have self-limited disease, and the course of their illness is not markedly different from that of Cryptosporidium enteritis in immunocompetent human immunodeficiency virus (HIV)-seronegative patients (13).

The diagnosis of cryptosporidial enteritis is based on identification of the oocyst in the stool. On routine ova and parasite examination their presence may be missed; consequently a modified acid-fast stain is used after concentration of the stool. Using these methods stool examination is positive in $70 \%$ to $80 \%$ of cases. Cryptosporidial organisms may also be identified in duodenal biopsy specimens because the organism lives in the brush border of intestinal epithelial cells throughout the gastrointestinal tract (15). Improvement in immune function with a concomitant increase in CD4 counts is the only way to eradicate cryptosporidial infection (13). Paromycin is being studied and has shown promise (16).

Microsporidium: Microsporidia represent another phylum of protozoa, comprising over 700 species. The most common organisms are Enterocytozoon bieneusi and Septata intestinalis. Microsporidia have been identified in 10\% to $30 \%$ of patients with AIDS and diarrhea. Prevalence rates vary depending on the biopsy site. Although the pathogenic role of E bieneusi has yet to be unequivocally established, it appears to cause a chronic diarrheal illness similar to that seen in cryptosporidiosis. Again the diarrhea is usually voluminous and nonbloody. Frequently patients also experience abdominal pain, flatulence and progressive weight loss despite maintaining a good appetite (17). Malabsorption is common and CD4 counts are typically less than 50 cells $/ \mathrm{mm}^{3}$. AIDS patients infected with microsporidia are most often homosexual and have a history of recent travel outside Canada and the United States.

The diagnosis of microsporidial infection is quite difficult. It is normally made by identification of spores in the stools, but concentration and staining techniques are cumbersome and of questionable accuracy. Electron microscopy is often necessary to make a definitive diagnosis of $E$ bieneusi $(15,17)$.
However, S intestinalis can often be found using light microscopy (18). The two organisms can also be differentiated based on location within the intestine; E bieneusi is located in the villus epithelial cells, while $S$ intestinalis is found in the lamina propria. Although there is no proven effective therapy to treat infections with these organisms, limited data suggest that microsporidia may be responsive to albendazole (SmithKline Beecham; emergency drug release only) (19). Unfortunately it appears that albendazole results in symptomatic improvement rather than eradication of the organism in patients with E bieneusi infections. More recent data suggest that microsporidia may respond to therapy with thalidomide (20). Thalidomide's true efficacy, however, awaits placebo controlled trials.

Isospora belli: Infection with Isospora belli can also cause chronic diarrhea in patients with AIDS. Although the organism is relatively uncommon in the United States (1\% to 3\%), it is a frequent cause of diarrhea in developing countries, where it occurs in $15 \%$ to $19 \%$ of patients with AIDS and diarrhea. I belli infects epithelial cells of the small intestine but can be identified throughout the entire gastrointestinal tract (15). Clinically, isosporiasis is similar to cryptosporidiosis. The diarrhea is generally watery and nonbloody and is associated with abdominal pain, anorexia, weight loss, fever and malaise. Unlike cryptosporidiosis, however, peripheral eosinophilia is found in up to $50 \%$ of patients infected by I belli (12).

Diagnosis of isosporiasis is established by either identification of oocysts in the stool or examination of small bowel biopsy specimens. Oocysts can be identified in the stool using a modified acid-fast stain. They are large (20 to $30 \mu \mathrm{m})$ and oval-shaped, differing from cryptosporidium both in size and number of sporoblasts. Biopsy specimens demonstrate altered mucosal architecture with villous atrophy and lymphocytic infiltration of the lamina propria. Organisms may be seen in the lumen or within cytoplasmic vacuoles in epithelial cells (15). Initial treatment with trimethoprim $(160 \mathrm{mg})$ and sulfamethoxazole $(800 \mathrm{mg}$ ) four times a day for 10 to 14 days has been shown to be effective in eradicating acute isospora infection (21). Pyrimethamine $75 \mathrm{mg}$ once a day is an effective alternative for those who cannot tolerate trimethoprim and sulfamethoxazole (22).

Other protozoa: A number of other protozoa have been identified in patients with AIDS and diarrhea, although they are predominantly found in homosexual men. Giardia lamblia, Entamoeba histolytica and Blastocystis hominis have been identified in the stools of up to $35 \%$ of homosexual men in selected populations (23). Clinically, giardiasis is the same in patients with AIDS as in immunocompetent individuals. E histolytica is a nonopportunistic pathogen that is rarely associated with diarrhea in AIDS patients. Unlike giardia, there is little evidence substantiating a pathogenic role for $B$ hominis (15). The treatment of these protozoa is the same in patients with AIDS as in immunocompetent individuals, ie, metronidazole $(24,25)$.

Viruses - Cytomegalovirus: Cytomegalovirus (CMV) is one of the most common and potentially serious opportunistic 
pathogens affecting patients with AIDS. Although CMV infection can occur anywhere within the gastrointestinal tract, the esophagus and colon are most frequently involved. The mechanism of infection is believed to be the result of reactivation of latent infection in immunocompromised individuals leading to $\mathrm{CMV}$ viremia and endothelial virus deposition. A vasculitis subsequently develops, leading to ischemia and ulcer formation. Symptomatic CMV enteritis occurs in $5 \%$ to $25 \%$ of AIDS patients. The most common clinical course is protracted watery diarrhea with associated lower abdominal cramping pain and fever, although there can be a broad spectrum of clinical presentations from intermittent diarrhea to severe hemorrhagic colitis or colonic perforation $(26,27)$.

Diagnosis of CMV colitis requires colonoscopy with biopsy, although endoscopic findings may vary. Discrete ulcers suggestive of early pseudomembranous colitis or more diffuse inflammatory changes (12) may be seen. Establishment of the diagnosis relies on the demonstration of CMV intranuclear inclusion bodies, in either epithelial or endothelial cells. These inclusion bodies are large, ovoid, intranuclear and surrounded by nuclear clearing. Serological studies or culture of urine or biopsy material alone are insufficient to establish the diagnosis since more than $90 \%$ of homosexual males with AIDS are seropositive for CMV (15). Recent randomized trials have demonstrated that gancyclovir is more effective than placebo in treating CMV colitis $(28,29)$. Unfortunately, the recurrence rate is significant and maintenance therapy with gancyclovir is often necessary (30). Foscarnet may be used in patients who do not respond to gancyclovir (31).

Herpes simplex virus: Herpes simplex virus is a well known cause of gastrointestinal disease, particularly in homosexual males, and can cause considerable morbidity in patients with AIDS. Herpes simplex infection in AIDS patients typically presents with perianal lesions, proctitis and/or esophagitis. Perianal lesions are chronic, cutaneous ulcers that may spread outside the perianal region if patients are particularly immunocompromised. Proctitis usually accompanies perianal disease and is manifested by severe anorectal pain, tenesmus, constipation and hematochezia. Endoscopically, it is nonspecific and most often localized to the distal $10 \mathrm{~cm}$ of the rectum. Individual lesions begin as small vesicles that progress to erosions and then coalesce into diffuse ulcers (12). Although herpes simplex virus is the leading cause of proctitis, it usually does not cause watery diarrhea.

Sigmoidoscopy is necessary to obtain biopsies and cytological brushings. Diagnosis is established based on cytological identification of intranuclear inclusion bodies in multinucleated cells from rectal ulcer brushings, and is confirmed by isolation of the virus from cultures of biopsy specimens (15). Acyclovir is the treatment of choice for herpes virus infections and can be administered topically, orally or intravenously. The optimal route depends on the location and severity of infection and therapy should be continued until all lesions have crusted or re-epithelialized. Recurrences occur frequently, often shortly after discontinuing acyclovir, suggesting the need for maintenance therapy (32).

Adenovirus: Adenovirus is a recognized cause of diarrhea in children and immunosuppressed adults without AIDS. Using electron microscopy, adenovirus has recently been identified in colonic tissue of $7 \%$ of AIDS patients with chronic, watery diarrhea (33). These patients often have associated weight loss without other identifiable enteric pathogens. Adenovirus appears to infect only mucosal cells, especially goblet cells, while sparing cells of the lamina propria. Ultrastructural evaluation reveals degeneration and focal necrosis of affected epithelial cells, suggesting that adenovirus may be pathogenic, although the virus may also be found in stool samples of patients without diarrhea. A causal relationship between adenovirus infection and diarrheal symptoms, however, remains to be established $(15,33)$.

Endoscopic evaluation of patients with adenovirus infection demonstrates either normal mucosa or raised, erythematous lesions measuring several millimetres. Light microscopy of biopsy specimens reveals chronic inflammation surrounding infected epithelial cells. These cells frequently contain intranuclear inclusion bodies which differ from CMV inclusions in that they occupy the entire nucleus (15). There is no effective treatment for adenovirus infections.

Other viruses: Several viruses have recently been identified as enteric pathogens in patients with T cell deficiency. Studies in patients with AIDS, however, have demonstrated conflicting results. Only the more common viruses such as rotavirus or adenovirus have been pursued and these agents have not consistently been associated with diarrhea. With improvements in isolation techniques, however, several novel viruses have recently been identified (34). Using these more sensitive methods, Grohmann and co-workers (34) found that the detection rate of viruses was actually higher than that of protozoa or bacteria, suggesting that viruses may be more common pathogens than previously suspected. Both astrovirus and calicivirus were found significantly more frequently in fecal specimens from patients with diarrhea than in specimens from patients without diarrhea, which suggests a potential causal role for these agents. It is not surprising that astrovirus was associated with diarrhea in immunocompromised patients because it is an established pathogen causing diarrhea in children. Similarly, rotavirus has been identified in the stools of AIDS patients with diarrhea and is believed to be a potential pathogen (35). Although picobirnaviruses were identified in $10 \%$ of patients with AIDS and diarrhea, the pathogenic influence of picobirnaviruses has not been established. Picobirnaviruses are small, bisegmented, doublestranded RNA viruses that were initially described in human feces in 1988 and subsequently identified in a number of animal hosts (34).

Bacteria - Mycobacterium: Gastrointestinal involvement with Mycobacterium avium complex (MAC) can occur alone but typically indicates systemic infection. Within the gastrointestinal tract, the small intestine seems to be more frequently involved than the colon. Large clusters of mycobacteria can be seen within macrophages of the small bowel 
lamina propria. Clinically, diarrhea and weight loss are common and malabsorption is present in more than $50 \%$ of affected patients. Although less common, colonic invasion results in chronic diarrhea and abdominal pain. The key host defect that allows MAC dissemination is thought to be macrophage dysfunction, since the mycobacteria are able to survive within macrophages for prolonged periods without being destroyed $(36,37)$.

Endoscopically, the small intestinal mucosa appears erythematous, edematous and friable, with small erosions and fine white nodules. Diagnosis of MAC infection is based on visualization of typical acid-fast organisms in stool or tissue $(23,37)$. Stool culture and acid-fast bacillus smear may be positive in up to $80 \%$ of infected patients. Blood culture is thought to be the most sensitive method to diagnose disseminated mycobacterial infection. Biopsies of the small bowel, lymph nodes or bone marrow are not as sensitive, but provide the advantage of rapid diagnosis because cultures take from one to six weeks to turn positive. Isolation and speciation, however, require culture of the organism.

In general, treatment of MAC has been disappointing because no medication, either alone or in combination, consistently eradicates the organism. A combination of amikacin, ethambutol, rifampin and ciprofloxacin has been shown to decrease bacterial load and improve symptoms significantly, but does not influence mortality (38). More recently, the combination of clarithromycin, rifabutin and ethambutol has shown promising results. Clarithromycin 1 to $2 \mathrm{~g}$ tid appears to be the most active agent, but is associated with the development of resistance when used as monotherapy. Therefore, combination therapy is still recommended using clarithromycin in combination with two of the following depending on in vitro sensitivity: rifabutin, ethambutol, ciprofloxacin, clofazimine or amikacin. The duration of therapy has not been established but likely needs to be indefinite (39).

Unfortunately, disseminated MAC infection eventually develops in most AIDS patients. In the absence of clearly effective treatment, more attention has been directed towards prophylaxis. Two placebo controlled trials of patients with AIDS and CD4 counts less than 200 cells $/ \mathrm{mm}^{3}$ demonstrated that prophylactic administration of rifabutin significantly reduced the frequency of disseminated MAC infection in this group (40). Based on these data, prophylaxis has been approved by the United States Food and Drug Administration and has become a common practice among HIV clinics $(39,40)$.

Other bacteria: Although the majority of serious infections in AIDS patients are due to protozoa or viruses, bacterial infections (Salmonella typhimurium, Salmonella enteritidis, Shigella flexneri, Campylobacter jejuni and Clostridium difficile) are also important. For example, AIDS patients demonstrate a 100-fold higher incidence of intestinal infection and associated bacteremia caused by salmonella compared with immunocompetent individuals. Moreover, infections are more prolonged or recurrent because of the loss of cellular immune function, antibiotic resistance or their combination $(15,23)$.
Cell-mediated immunity is important in controlling salmonella infections since the organism can live intracellularly for a long time despite humoral immunity or antibiotics. By contrast, shigella, $\mathrm{C}$ jejuni and $\mathrm{C}$ difficile do not appear to be more virulent in patients with AIDS.

Clinically, patients with salmonellosis present with watery, nonbloody diarrhea associated with intermittent fever. When diarrhea is present, it is usually severe and may be associated with bloating and nausea. In contrast, infection with shigella or campylobacter is often associated with severe abdominal cramping, tenesmus and the frequent passage of bloody stools (23). Diagnosis of salmonella infection is established by stool culture, although blood cultures are frequently positive. Stool examination may show fecal leukocytes, but the sensitivity and specificity of this test in AIDS patients with salmonellosis has not been established. Fecal leukocytes are more commonly found in stool specimens from patients with shigella or campylobacter infection because these organisms are invasive $(23,41,42)$. Treatment options for eradication of bacterial enteritides in patients with AIDS include intravenous ampicillin, trimethoprim-sulfamethoxazole, third-generation cephalosporins and ciprofloxacin. Selection of specific antibiotics, however, should be guided by in vitro sensitivity testing $(30,41-43)$. Even though these infections typically respond to antibiotic therapy they frequently recur. Furthermore, AIDS patients seem to be uniquely susceptible to recurrent episodes of salmonella bacteremia; consequently suppressive therapy is generally recommended. Oral ciprofloxacin $750 \mathrm{mg}$ tid has been found to be successful in both clearing the initial bacteremia and acting as suppressive therapy (44). Metronidazole and vancomycin appear to be equally effective in treating $\mathrm{C}$ difficile enterocolitis (45).

HIV enteropathy: From $20 \%$ to $50 \%$ of AIDS patients with diarrhea have no identifiable pathogen (46) and may have 'AIDS enteropathy'. This syndrome is characterized by diarrhea, malabsorption and weight loss. Histological examination of intestinal biopsies reveals a triad of partial villous atrophy, crypt hyperplasia and mild to moderate inflammation (47).

Several theories have been proposed to explain these observations $(15,17)$. One hypothesis is that opportunistic agents causing diarrhea are either missed or have not yet been identified as pathogens. This theory is supported by identification of new parasites such as E bieneusi and several new viruses using electron microscopy and more sensitive isolation techniques, respectively (37). Bacterial overgrowth is another potential explanation for malabsorption and concomitant diarrhea. Bacterial overgrowth is thought to result from a combination of diminished immunoglobulin production, reduced acid secretion and altered intestinal motility (15).

Considerable evidence suggests that HIV may cause diarrhea. HIV is commonly identified in the lamina propria of the gastrointestinal tract of AIDS patients. The presence of HIV in the gastrointestinal tract has been observed using polymerase chain reaction techniques, RNA and DNA in 


\section{TABLE 1}

Effective agents for the eradication of specific opportunistic infections

\begin{tabular}{|c|c|c|}
\hline Organism & Primary treatment (reference) & Secondary treatment (reference) \\
\hline \multicolumn{3}{|l|}{ Protozoa } \\
\hline Cryptosporidium & Paromycin $500-750 \mathrm{mg}$ tid $\times 10$ days $(16)^{*}$ & \\
\hline Microsporidia & Albendazole $400 \mathrm{mg}$ bid $\times 28$ days $(19)^{*}$ & Thalidomide $100 \mathrm{mg}$ at night $\times 21$ days $(20)^{*}$ \\
\hline Giardia lamblia & Metronidazole $250 \mathrm{mg}$ tid $\times 7$ days (25) & Quinacrine $100 \mathrm{mg}$ tid $\times 5$ days (24) \\
\hline Entamoeba histolytica & Metronidazole 750 mg tid x 10 days (25) & lodoquinol $650 \mathrm{mg}$ tid $\times 20$ days (25) \\
\hline Cytomegalovirus & Gancyclovir 5 mg/kg bid IV x 14 days (28) & Foscarnet 30 mg/kg tid IV (31) \\
\hline Herpes virus & Acyclovir $15-30$ mg/kg/day IV (32) & \\
\hline Adenovirus & None & \\
\hline \multicolumn{3}{|l|}{ Bacteria } \\
\hline Salmonella species & Amoxicillin $1 \mathrm{~g}$ tid $\times 14$ days (43) & Ciprofloxacin $500 \mathrm{mg}$ bid $\mathrm{x} 7$ days (15) \\
\hline
\end{tabular}

situ hybridization, immunohistological studies using monoclonal antibodies to HIV-associated protein antigens, and antigen capture ELISA (48). It is hypothesized that infection of lamina propria cells results in structural and functional changes in the small bowel mucosa that leads to malabsorption and diarrhea. Ulrich and colleagues (49) identified a large percentage of HIV-infected patients with decreased or absent lactase activity in the brush border membrane. Moreover, they identified an enterocyte maturational defect, or 'hyporegeneration', that was most pronounced in patients demonstrating HIV antigen p24 in the mucosa (49). By contrast, AIDS patients treated with zidovudine were less likely to be lactase deficient and demonstrated a more normal maturation of enterocytes (17). HIV may lead to diarrhea via other mechanisms as well. Infection of the lamina propria may result in a deficiency of mucosal trophic factors or damage by enteric organisms. Bigornia et al (50) identified HIV-1 protein and nucleic acid in enterochromaffin cells of patients with diarrhea, but not in patients without diarrhea, suggesting that the virus may alter the function of the enterochromaffin cells and lead to diarrhea (50).

HIV infection may also lead to diarrhea indirectly via its influence on gut immunology. CD4 T cell abnormalities in both the peripheral blood and gut mucosa have been well described in patients with AIDS. Abnormal activation of epithelial lymphocytes and deficiency of mast cells may lead to an inflammatory reaction similar to that observed in acute graft-versus-host disease. Recent work by Kotler et al (48) supports the theory of an immunological defect leading to diarrhea. These researchers demonstrated an association between HIV antigen expression and inflammatory disease of the intestinal mucosa in patients with or without AIDS. Tissue concentrations of inflammatory mediators

\author{
TABLE 2 \\ Potential agents for use in the symptomatic treatment of \\ acquired immunodeficiency syndrome-related diarrhea

\begin{tabular}{l} 
Antimotility agents \\
Diphenoxylate with atropine \\
Loperamide \\
Codeine phosphate \\
Morphine sulphate \\
Methadone \\
Camphorized tincture of opium \\
Deodorized tincture of opium \\
Luminal acting agents \\
Dietary fibre \\
Cholestyramine \\
Sucralfate \\
Hormonal therapy \\
Octreotide - dosage: $50-500 \mu g$ subcutaneously tid \\
\hline
\end{tabular}

were higher in HIV-infected and AIDS patients than in controls. Furthermore, they observed an interrelationship among mucosal HIV p24 antigen, altered bowel function and mucosal inflammation which was independent of the presence of enteric pathogens (48). Although there is substantial evidence supporting the pathogenic role of HIV in AIDS enteropathy, no study has been able to establish a cause and effect relationship. Thus, the exact mechanism(s) whereby HIV exerts its effects require further clarification.

\section{THERAPY}

Specific treatment: Analysis of the literature regarding treatment of specific enteric pathogens in AIDS patients reveals a paucity of randomized clinical trials. In most cases the 
specific therapy does not differ from that used in other patient populations. However, bacterial organisms isolated from AIDS patients may be more resistant to treatment with antibiotics than the same organisms isolated from immunocompetent individuals. Moreover, these organisms typically recur and require chronic suppressive therapy; thus AIDS patients may be predisposed to developing infections with drug-resistant organisms $(15,30)$. Various therapeutic agents with proven efficacy in the treatment of specific opportunistic enteric infections are listed in Table 1.

Symptomatic treatment: Depending on the study, from 20\% to $50 \%$ of patients may have no identifiable pathogen despite an extensive evaluation (46). Furthermore, there is no proven effective therapy for several clinically important pathogens such as cryptosporidium, microsporidium and MAC. Thus, symptomatic therapy remains an important part of the management of diarrhea in AIDS patients. There are three main categories of nonspecific antidiarrheal therapy: antimotility agents, luminal acting agents and hormonal therapy (Table 2) (30).

As their name implies, antimotility agents function by decreasing intestinal transit. Narcotic analgesics such as codeine, morphine sulphate and tincture of opium are effective antimotility agents, but are not widely used because of their addictive properties. Loperamide and diphenoxylate are nonaddicting and are commonly used for symptomatic control of diarrhea. Although there are anecdotal reports of symptomatic improvement in cryptosporidial diarrhea (51), there are no randomized, controlled clinical trials establishing the efficacy of antimotility agents in patients with AIDS-related diarrhea. The maximum recommended dose of either loperamide or diphenoxylate is eight tablets or capsules per day, but higher doses may be necessary in patients with severe diarrhea. Luminal acting agents, the most common of which is fibre, function by absorbing excess fluid in the intestine. Fibre ingestion results in increased stool bulk with concomitant prolongation of transit time. Similarly, cholestyramine, an ion exchange resin, reduces diarrhea by binding bile acids. Neither fibre nor cholestyramine is particularly effective in patients with severe diarrhea (30).

Octreotide, a synthetic analogue of somatostatin, has received increasing attention as a potential treatment for AIDS-related diarrhea. Administered subcutaneously, octreotide inhibits all gastrointestinal hormones, resulting in increased transit time, diminished fluid secretion and increased absorption of fluids and electrolytes. Anecdotal case reports have demonstrated improvement in diarrhea associated with octreotide therapy (52-54). Moreover, three prospective but uncontrolled trials have demonstrated response rates between $45 \%$ and $66 \%$ using octreotide in doses ranging between $50 \mu \mathrm{g}$ and $500 \mu \mathrm{g}$ tid. In all studies the diarrhea recurred upon cessation of treatment, although it again improved when octreotide was restarted (55-57). Despite these initial encouraging results, a randomized, placebo controlled trial did not establish similar efficacy (58). In a multicentre study, Simon and colleagues (58) compared octreotide (in doses up to $300 \mu \mathrm{g}$ tid) with placebo in 129 patients with more than $500 \mathrm{~g}$ of stool daily. After three weeks no significant difference was observed in response rates between octreotide and placebo groups. Based on the results of this multicentre study, it is recommended that octreotide be used only in cases of refractory AIDS diarrhea after maximum tolerated doses of standard antidiarrheal agents have failed (Table 2) (58).

\section{MANAGEMENT STRATEGY}

The diagnosis and treatment of patients with HIV-related diarrhea remain controversial. Most experts recommend an extensive evaluation with specific treatment of all pathogens. However, a recent decision analysis comparing various management strategies questioned the necessity of an extensive evaluation (59). The results of this decision analysis revealed that a more limited evaluation (stool studies and blood cultures) with symptomatic therapy given to patients in whom no specific pathogen was identified was as effective as an initial extensive evaluation in managing the diarrhea. The similarity of response rates was probably due to the lack of effective treatment options for pathogens such as cryptosporidium, microsporidium and MAC, which are typically identified by extensive evaluation (59). Although these strategies may seem disparate, they are quite similar. The main difference is a philosophical one. Those who recommend an extensive evaluation do not treat until a specific pathogen is identified. Symptomatic therapy is reserved for only the subset of patients in whom no pathogen can be identified. By contrast, the limited approach advocates the use of initial symptomatic therapy if stool studies are negative. If there is no response to symptomatic therapy, patients then undergo further evaluation including endoscopic studies.

The challenge confronting physicians who care for HIV infected patients with diarrhea is to determine the most appropriate management strategy. For each affected individual the main focus should be to determine the likelihood that the planned evaluation will lead to an improvement in the patient's outcome $(60,61)$. The answer will probably differ depending on the clinical situation. An extensive evaluation may not be justified in all cases. Similarly, a limited evaluation is not appropriate in all situations. An initial extensive evaluation seems reasonable if experimental treatment protocols are available and easily accessible. If not, it may be more appropriate to perform a limited examination including stool studies (stool culture, ova and parasites examination, and protozoan stains) and blood cultures. If no specific pathogen is identified, then the patient can be treated symptomatically. Endoscopic studies should be reserved for those who did not respond to symptomatic treatment. The need for further investigation into the pathogenesis of AIDS-related diarrhea is well recognized. There is also a need for additional research evaluating the effects of various management strategies on the frequency and severity of diarrhea, as well as on broader outcome measures such as functional status and quality of life. 


\section{REFERENCES}

1. Santangelo WC, Krejs GJ. Southwestern Internal Medicine Conference: gastrointestinal manifestations of the acquired immunodeficiency syndrome. Am J Med Sci 1986;292:328-34.

2. Friedman SL, Owen RL. Gastrointestinal manifestations of AIDS and other sexually transmitted diseases. In: Sleisenger MH, Fordtran JS, eds. Gastrointestinal Disease: Pathophysiology, Diagnosis and Management. Philadelphia: WB Saunders Company, 1988:1242-77.

3. Antony MA, Brandt LJH, Klein RS, Bernstein SLH. Infectious diarrhea in patients with AIDS. Dig Dis Sci 1988;33:1141-6.

4. Dworkin B, Wormser GP, Rosenthal WS, et al. Gastrointestinal manifestations of the acquired immunodeficiency syndrome: a review of 22 cases. Am J Gastroenterol 1985;80:774-8.

5. Gelb A, Miller S. AIDS and gastroenterology. Am J Gastroenterol 1986;81:619-22.

6. Rolston KVI, Rodriguez S, Hernandez M, Bodey GP. Diarrhea in patients infected with the human immunodeficiency virus. Am J Med 1989;86:137-8.

7. René E, Marche C, Regnier B, et al. Intestinal infections in patients with acquired immunodeficiency syndrome. Dig Dis Sci 1989;34:773-80.

8. Smith PD, Lane HC, Gill VJ, et al. Intestinal infections in patients with the acquired immunodeficiency syndrome: etiology and response to therapy. Ann Intern Med 1988;108:328-33.

9. Gillin JS, Shike M, Alcock N, et al. Malabsorption and mucosal abnormalities of the small intestine in the acquired immunodeficiency syndrome. Ann Intern Med 1985;102:619-22.

10. Laughon BE, Druckman DA, Vernon A, et al. Prevalence of enteric pathogens in homosexual men with and without acquired immunodeficiency syndrome. Gastroenterology 1988;94:984-93.

11. Rabenek L, Crane MM, Risser JMH, Lacke CE, Wray NP. Effect of HIV transmission category and CD4 count on the occurrence of diarrhea in HIV-infected patients. Am J Gastroenterol 1993;88:1720-3.

12. Rodgers VD, Kagnoff MF. Gastrointestinal manifestations of the acquired immunodeficiency syndrome. West J Med 1987;146:57-67.

13. Flanigan $T$, Whalen $C$, Turner J, et al. Cryptosporidium infection and CD4 counts. Ann Intern Med 1992;116:840-2.

14. Navin TR, Juranek DD. Cryptosporidiosis: clinical, epidemiologic, and parasitic review. Rev Infect Dis 1984;6:313-27.

15. Smith PD, Quinn TC, Strober W, Janoff EN, Masur H. NIH Conference: gastrointestinal infections in AIDS. Ann Intern Med 1992;116:63-77.

16. Armitage K, Flanigan T, Carey J, et al. Treatment of cryptosporidiosis with paromycin. Arch Intern Med 1992;152:2497-9.

17. Simon D, Brandt LJ. Diarrhea in patients with the acquired immunodeficiency syndrome. Gastroenterology 1993;105:1238-42.

18. Rijpstra AC, Canning EU, Van Ketel RJ, Eeftinck Schattenkerk JKM, Laarman JJ. Use of light microscopy to diagnose small-intestinal microsporidiosis in patients with AIDS. J Infect Dis 1988;157:827-31.

19. Blanshard C, Ellis DS, Tovey DG, Dowell S, Gazzard BG. Treatment of intestinal microsporidiosis with albendazole in patients with AIDS. AIDS 1992;6:311-3.

20. Sharpstone D, Rowbottom A, Nelson M, Gazzard B. The treatment of microsporidial diarrhea with thalidomide. AIDS 1995;9:658-9.

21. Pape JW, Verdier RI, Johnson WD. Treatment and prophylaxis of Isospora belli infection in patients with the acquired immunodeficiency syndrome. N Engl J Med 1989;321:1044-7.

22. Weiss LM, Perlman DC, Sherman J, et al. Isospora belli infection: treatment with pyrimethamine. Ann Intern Med 1988;109:474-5.

23. Lopez AP, Gorbach SL. Diarrhea in AIDS. Infect Dis Clin 1988;2:705-19.

24. Wolfe MS. Current concepts in parasitology, giardiasis. N Engl J Med 1978;298:319.

25. Drugs for parasitic infections. Med Lett 1990;32:23-32.

26. René E, Marche C, Chevalier T, et al. Cytomegalovirus colitis in patients with acquired immunodeficiency syndrome. Dig Dis Sci 1988;33:741-50.

27. Dieterich DT, Rahmin M. Cytomegalovirus colitis in AIDS: presentation in 44 patients and a review of the literature. J Acquir Immune Defic Syndr 1991:4(Suppl 1):S29-35.

28. Chachoua A, Dieterich D, Krasinski K, et al. 9-(1,3-dihydroxy-2-propoxymethyl) guanine (gancyclovir) in the treatment of cytomegalovirus gastrointestinal disease with the acquired immunodeficiency syndrome. Ann Intern Med 1987;107:133-7.
29. Dieterich DT, Kotler DP, Busch DF, et al. Gancyclovir treatment of cytomegalovirus colitis in AIDS: a randomized, double-blind, placebo-controlled multicenter study. J Infect Dis 1993;167:278-82.

30. Simon D, Weiss LM, Brandt LJ. Treatment options for AIDS-related esophageal and diarrheal disorders. Am J Gastroenterol 1992;87:274-81.

31. Dieterich DT, Poles MA, Dicker M, Tepper R, Lew E. Foscarnet treatment of cytomegalovirus gastrointestinal infections in acquired immunodeficiency syndrome patients who have failed gancyclovir induction. Am J Gastroenterol 1993;88:542-8.

32. Drew WL, Buhles W, Erlich KS. Herpesvirus infections: how to use gancyclovir and acyclovir. Infect Dis Clin 1988;2:495-509.

33. Janoff EN, Orenstein JM, Manischewitz JF, Smith PD. Adenovirus colitis in the acquired immunodeficiency syndrome. Gastroenterology 1991;100:976-9.

34. Grohmann GS, Glass RI, Pereira HG, et al. Enteric viruses and diarrhea in HIV-infected patients. N Engl J Med 1993;329:14-20.

35. Cunningham AL, Grohman GS, Harkness J, et al. Gastrointestinal viral infections in homosexual men who were symptomatic and seropositive for human immunodeficiency virus. J Infect Dis 1988;158:386-91.

36. Jacobson MA. Mycobacterial diseases. Infect Dis Clin 1988;2:465-74.

37. Hawkins CC, Gold JWM, Whimbey E, et al. Mycobacterium avium complex infections in patients with the acquired immunodeficiency syndrome. Ann Intern Med 1986;105:184-8.

38. Chiu J, Nussbaum J, Bozzette S, et al. Treatment of disseminated Mycobacterium avium complex infection in AIDS with amikacin, ethambutol, rifampin, and ciprofloxacin. Ann Intern Med 1990;113:358-61.

39. Smith GH. Treatment of infections in patients with acquired immunodeficiency syndrome. Arch Intern Med 1994;154:949-73.

40. Nightingale SD, Cameron DW, Gordin FM, et al. Two controlled trials of rifabutin prophylaxis against Mycobacterium avium complex infections in AIDS. N Engl J Med 1993;329:828-33.

41. Baskin DH, Lax JD, Barenberg D. Shigella bacteremia in patients with the acquired immunodeficiency syndrome. Am J Gastroenterol 1987;82:338-41.

42. Bernard E, Roger PM, Carles D, et al. Diarrhea and campylobacter infections in patients infected with human immunodeficiency virus. J Infect Dis 1989;159:143-4.

43. Jacobs JL, Gold JWM, Murray HW, et al. Salmonella infections in patients with the acquired immunodeficiency syndrome. Ann Intern Med 1985;102:186-8.

44. Chaisson RE. Infections due to encapsulated bacteria, salmonella, shigella, and campylobacter. Infect Dis Clin North Am 1988;2:475-84.

45. Bartlett J. Clostridium difficile: clinical observations. Rev Infect Dis 1990;12:S243-51.

46. Greenson JK, Belitsos PC, Yardley JH, Bartlett JG. AIDS enteropathy: occult enteric infections and duodenal mucosal alterations in chronic diarrhea. Ann Intern Med 1991;114:366-72.

47. Kotler DP, Gaetz HP, Lange M, Klein EB, Holt PR. Enteropathy associated with the acquired immunodeficiency syndrome. Ann Intern Med 1984;101:421-8.

48. Kotler DP, Reka S, Clayton F. Intestinal mucosal inflammation associated with human immunodeficiency virus infection. Dig Dis Sci 1993;38:1119-27.

49. Ulrich R, Zeitz M, Heise W, L'age M, Höffken G, Rieken EO. Small intestine structure and function in patients infected with human immunodeficiency virus (HIV): evidence for HIV-induced enteropathy. Ann Intern Med 1989;111:15-21.

50. Bigornia E, Simon D, Weiss LM, et al. Detection of HIV-1 protein and nucleic acid in enterochromaffin cells of HIV-1 seropositive patients. Am J Gastroenterol 1992;87:1624-8.

51. Connolly GM, Dryden MS, Shanson DC, Gazzard BG. Cryptosporidial diarrhea in AIDS and its treatment. Gut 1988;29:593-7.

52. Robinson EN, Fogel R. SMS 201-995, a somatostatin analogue, and diarrhea in the acquired immunodeficiency syndrome (AIDS). Ann Intern Med 1988;109:680-1.

53. Cook DJ, Kelton JG, Stanisz AJM, Collins SM. Somatostatin treatment for cryptosporidial diarrhea in a patient with the acquired immunodeficiency syndrome (AIDS). Ann Intern Med 1988;108:708-9. 
54. Simon D, Weiss L, Tanowitz HB, Wittner M. Resolution of cryptosporidium infection in an AIDS patient after improvement of nutritional and immune status with octreotide. Am J Gastroenterol 1991;86:615-8.

55. Cello J, Grendal J, Basu P, et al. Effect of octreotide on refractory AIDS-associated diarrhea. Ann Intern Med 1991;115:705-10.

56. Fanning M, Monte M, Sutherland LR. Pilot study of sandostatin (octreotide) therapy of refractory HIV-associated diarrhea. Dig Dis Sci 1991;36:476-80.

57. Moroni M, Franzetti F, Carosi GP, et al. Effect of octreotide treatment in patients with refractory AIDS-associated diarrhea. Gastroenterology 1991;100:A601.
58. Simon DM, Cello JP, Valenzuela J, et al. Multicenter trial of octreotide in patients with refractory acquired immunodeficiency syndrome-associated diarrhea. Gastroenterology 1995;108:1753-60.

59. Johanson JF, Sonnenberg A. Efficient management of diarrhea in the acquired immunodeficiency syndrome (AIDS). Ann Intern Med 1990;112:942-8

60. Brady CE. AIDS-related diarrhea: what's a gastroenterologist to do? Am J Gastroenterol 1991;86:1685-6.

61. Rabenek L. Diagnostic workup strategies for patients with HIV-related chronic diarrhea. J Clin Gastroenterol 1993;16:245-50. 


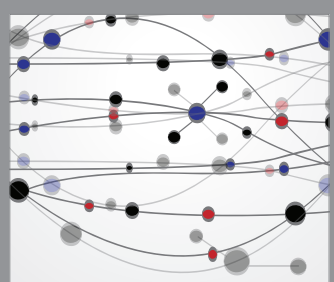

The Scientific World Journal
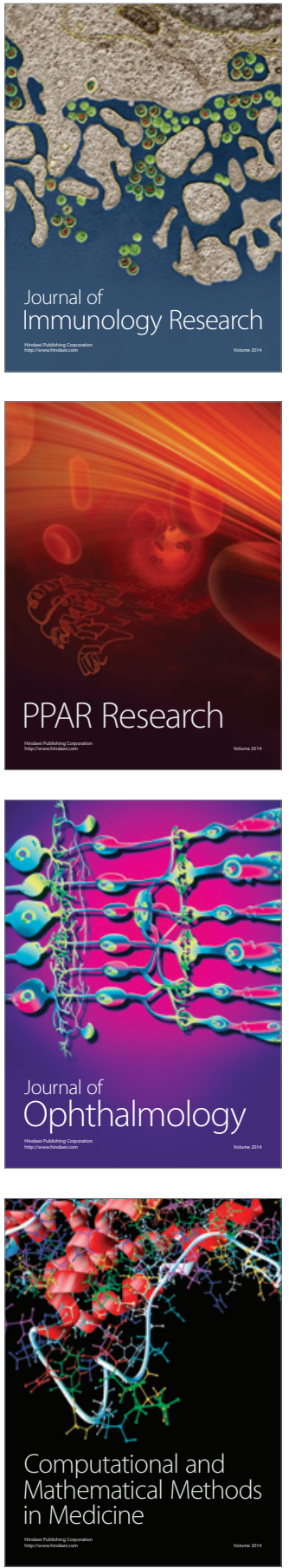

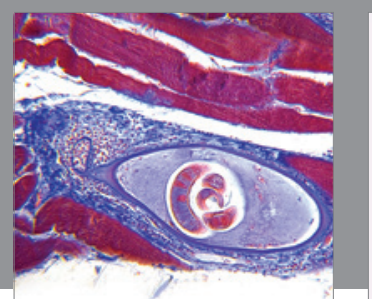

Gastroenterology Research and Practice

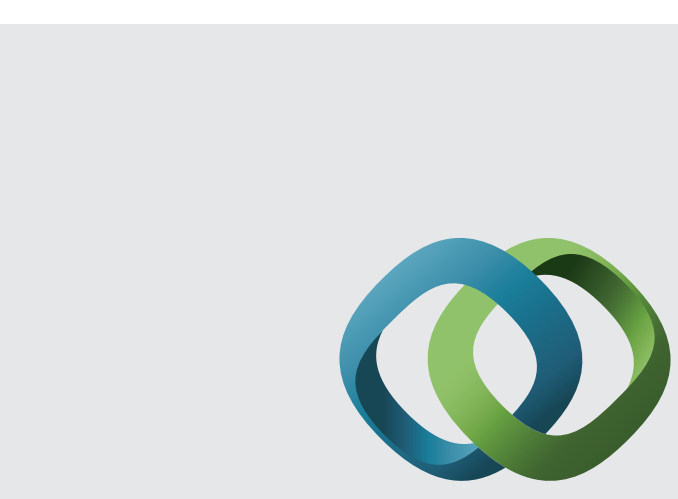

\section{Hindawi}

Submit your manuscripts at

http://www.hindawi.com
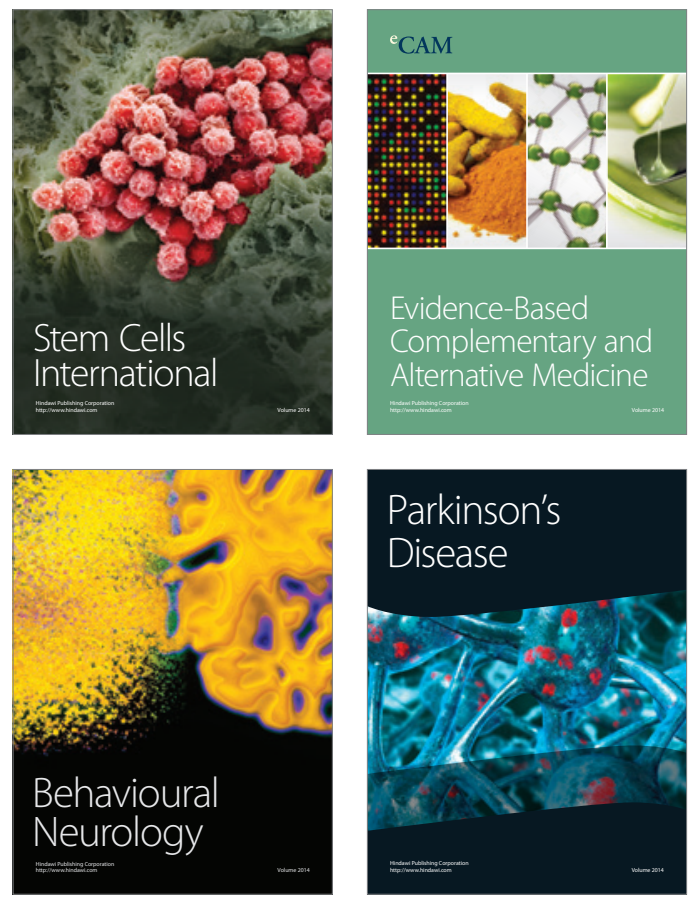
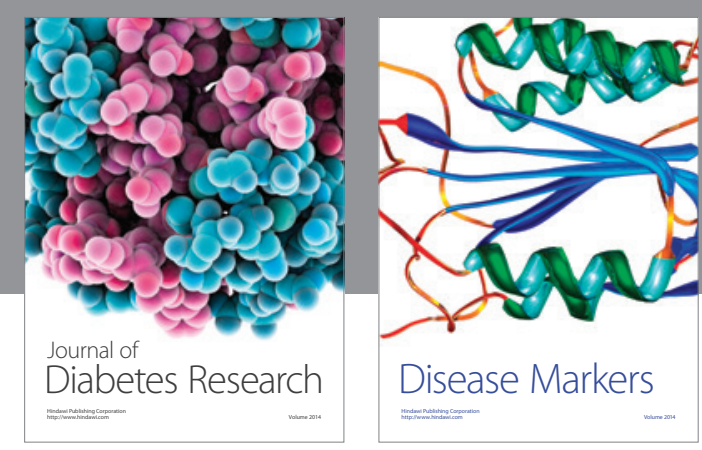

Disease Markers
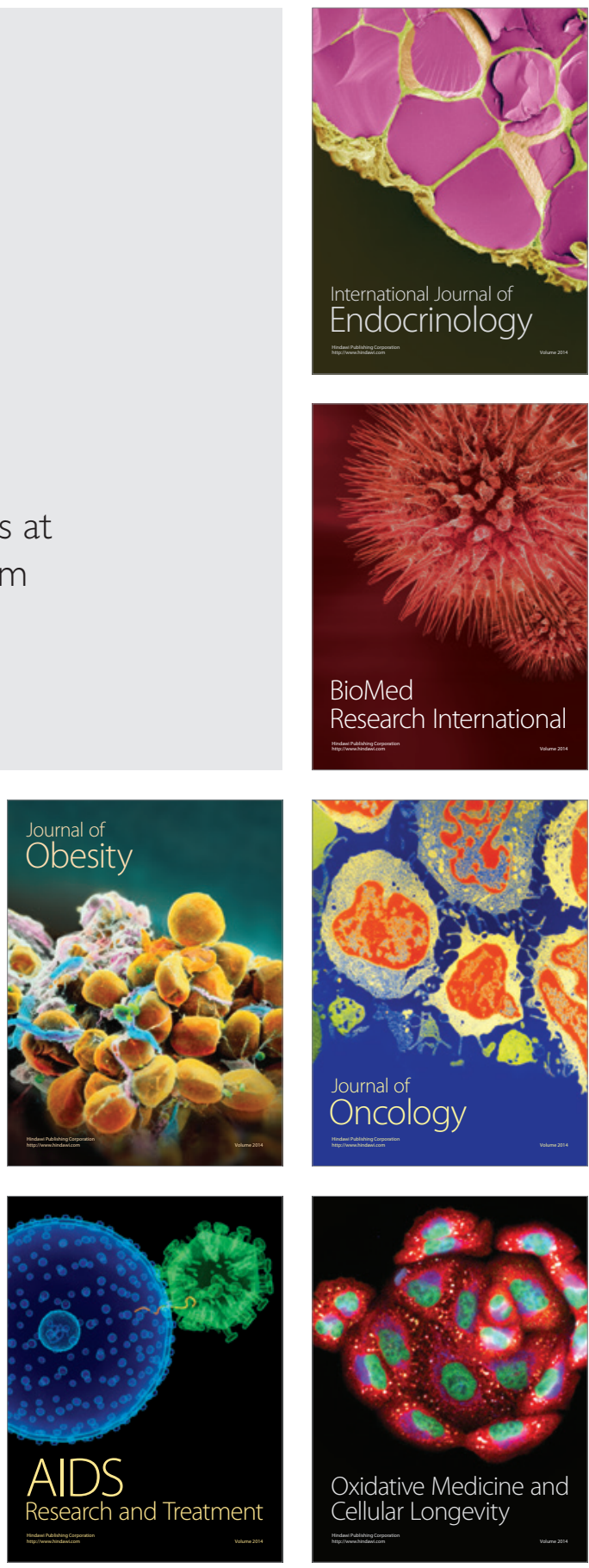